\title{
Human-Centric Functional Modeling of Biocomputers
}

Andy E. Williams, Nobeah Foundation, Nairobi, Kenya

\begin{abstract}
This paper explores how the technique of Human-Centric Functional Modeling might potentially be used to represent a broad subset of proposed implementations of biocomputing with anywhere from narrow to general problem-solving ability within a given domain, or across multiple domains, and how such functional models might be implemented by libraries of biological computing mechanisms. This paper also explores the insights to be gained from modeling biocomputers this way, and how HumanCentric Functional Modeling might significantly accelerate research and increase the impact of research in biocomputing through significantly increasing capacity for reuse of both biocomputing hardware and software.
\end{abstract}

Keywords: Human-Centric Functional Modelling, biocomputing, General Collective Intelligence

\section{Background}

In Human-Centric Functional Modeling living systems are modeled as having a set of behaviours (functions). All the states accessible through these functions within a given domain of behaviour form a "functional state space" which the system moves through as in figure 1. From this perspective, cognition can be modeled as moving through a space in which the states are concepts (a "conceptual space").

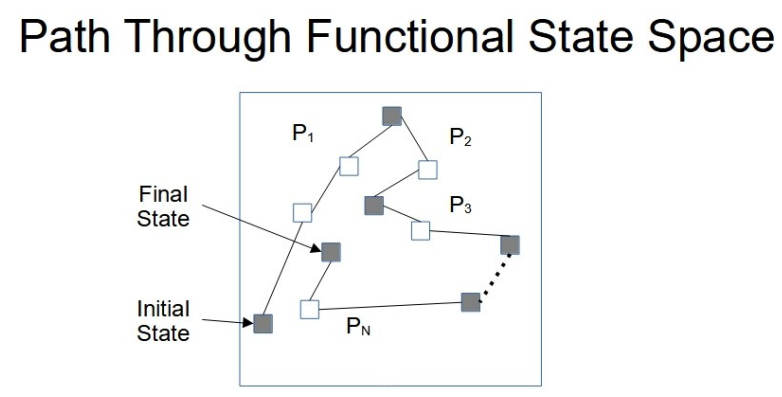

Figure 1: In Human-Centric Functional Modeling systems are represented as moving through a functional state space.

Representing general problem-solving ability in any domain as solving the problem of navigating from any one functional state in that domain to potentially any other, then if some minimal set of functions (operations) exists that can be used to compose all other functions in a given domain, all processes that solve any problem in a given domain can be represented in terms of that minimal set. Viewing problem solving processes in a given domain as "computing" processes in that domain, then that set of functions can represent any computational process connecting functional states. The importance of this is that having potentially created the capacity to represent all computational processes that might be used to navigate between functional states, then from the functional modelling perspective biological systems 
can then be represented as some process of computation which achieves navigation between those states. For example, from the functional modelling perspective the cognition can be seen as navigating a conceptual space. Solving the problem of achieving general problem-solving ability in the cognitive domain requires navigating the conceptual space with general problem solving ability, that is, with the ability to potentially navigate from any general concept defining the problem, to any general concept defining the solution.

Any system with a stable set of repeatable functions also must stay within a bounded region of a "fitness space" that describes the fitness of the system to execute its functions. Defining a "fitness space" in terms of target value of fitness, actual value of fitness, and predicted value of fitness of the system that is achieved as a result of some action (some path) in functional state space, the path through this fitness space must stay within a bounded region in fitness space. In this sense the motion in fitness space must be stable globally throughout the fitness space, despite potentially being chaotic in functional state space due to random interactions with the environment as in figure 2.

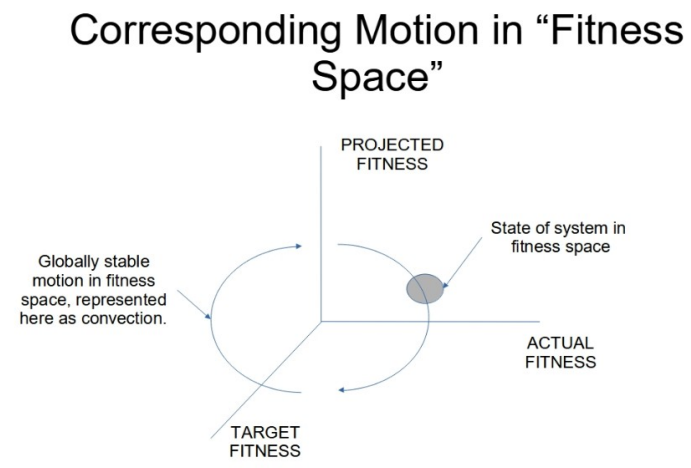

Figure 2: In Human-Centric Functional Modeling systems are represented as also moving through a fitness space as they move through functional state space.

All the processes of life, beginning with homeostasis, and ending in consciousness, and cognition, can potentially be modelled in this same way as processes with this general problem-solving ability, namely as processes that adapt to solve any general problem of navigation within their functional domain in order to solve the problem of maintaining dynamic stability in their fitness space. This is general problem-solving ability in each domain that non-living systems of computation might not have.

Representing each process as an adaptive process that operates within a particular adaptive domain, and representing each process as being implemented by a functional component, life is then a hierarchy of adaptive processes implemented by a hierarchy of functional components as in figure 3 . 


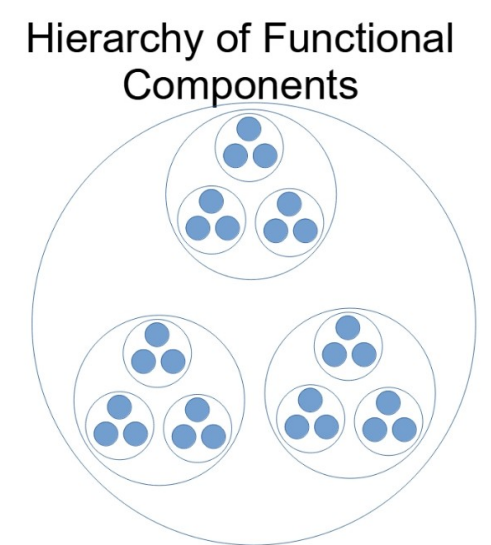

Figure 3: In Human-Centric Functional Modeling living systems are represented in terms of a hierarchy of processes and the hierarchy of functional components that implement those processes. Processes lower on the hierarchy represented as circles are depicted as being enclosed (occurring within) processes higher up on the hierarchy represented as larger circles.

Any such adaptive process operates within a functional state space in which each function is implemented by a child functional component. Considering processes to be implemented by a parent functional component, then that parent functional component will have child functional components which implement those functions as in figure 4.

\section{Functional Components}

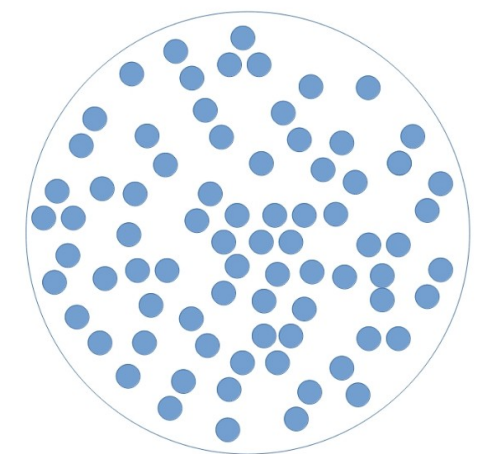

Figure 4: In Human-Centric Functional Modeling systems are represented as having a set of functional components that implement all their functions.

This set of child functional components collectively travels through the functional state space of the parent functional component they are contained within, and the parent functional component will travel through the collective functional state space of its child functional components. The parent, as a collection of functional components, will travel through that functional state space according to the dynamics required for stability within its fitness space.

Using a swim lane diagram, the process of the collection of functional components navigating the collective functional state space can be represented as $\mathrm{N}$ functional components each executing an activity within the collective process as in figure 5. 


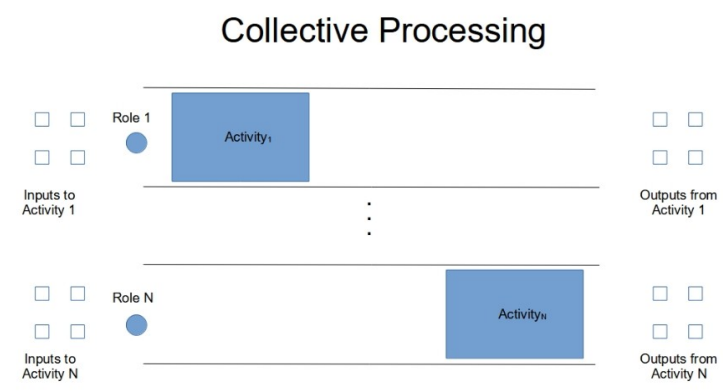

Figure 5: In Human-Centric Functional Modeling systems executing processes are represented as executing a sequence of functional components, each of which executes an activity in that process.

In addition, the swim lane diagram clarifies that outputs from one activity conducted by one functional component are inputs to another activity conducted by another functional component. In order for this interaction between functional components to take place, and in order for this optimization function to be executed, there must be a network of channels between functional components. The more it is true that any functional component can play any role, the more heavily interconnected this network must be. As a result of the need for a heavily interconnected network as in figure 6 as well as the need for other required functionality through which a decentralized collective optimization processes might be executed, without these requirements it is proposed here that collective optimization problems cannot reliably be solved.

\section{Channels Between Functional Components}

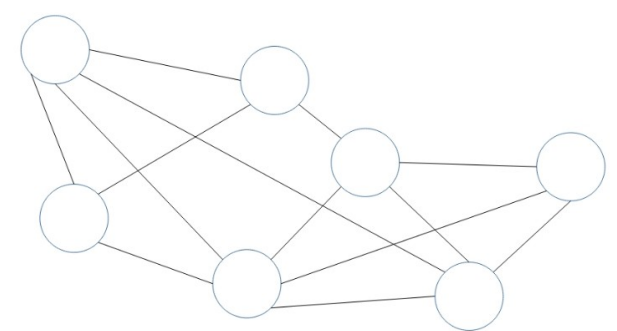

Figure 6: In Human-Centric Functional Modeling systems execute processes by executing a sequence of functional components and must define channels to exchange inputs and outputs between these functional components.

While such functional models ignore all details of the physical processes and structures through which the functions are actually implemented, a Human-Centric Functional Modeling approach is potentially invaluable in understanding those implementation mechanisms. By defining a single common functional model of living organisms, in which every functional component is a biocomputer that computes outcomes in some domain, every functional component becomes a category for storing the knowledge of any researcher about that function, and every mechanism by which that function is proposed to be implemented becomes part of a library of potential implementation mechanisms. If all potential biological functions can be represented as occurring within some functional domain in the HumanCentric Modeling Approach, then all detailed models for all biological structures having such functions can be represented as part of a library of implementations for some functional component in such a functional model. Through defining a mechanism for evaluating the "fitness" of each implementation in representing observed behaviour, all research into all functions achieve by all biological structures might reliably converge on a single understanding that is most "fit". 
A recently developed model of Artificial General Intelligence (AGI) is one example of this approach to modeling biological computing [1]. In this approach, the orchestration of cooperation between functional components by some collective optimization function applies whether talking about functional components in an individual cognition, or individual humans in a collective cognition. Using this insight, a model for collaborative computing called General Collective Intelligence (GCI) was developed as another example of biologically inspired computing [2]. As with all such Human-Centric Functional Models, the model of AGI creates the potential to define functional models of all existing AI approaches and solutions, and to use that library of implementations to increase the general problem-solving ability of the AGI [3]. Similarly, the model of GCI creates the potential to define functional models of all existing Collective Intelligence (CI) solutions, so they might be combined into a library the GCI can use to increase its general problem-solving ability [4].

Problems that require collective reasoning are those that span a much larger region of the collective conceptual space, and do so at much higher resolution, than can reliably be navigated by any individual human cognition. Any problem that requires the ability to query and incorporate any general information about all individuals in the group is one that might require collective reasoning.

\section{Introduction}

From the time Leonard Adleman showed the feasibility of implementing human-defined computations with molecular (i.e., genetic) hardware [7], the field of biocomputing has expanded to contain a wide range of approaches. These approaches are defined according to a number of categories introduced and defined by their researchers. One category introduced and defined is 'heterotic computing', a combination of two or more computational systems such that they provide an advantage over either substrate used separately [8].

Others propose to group the processing of information by cells into two types of computing (i.e., genetic and metabolic) depending on the nature of the input and components thereof, in order to highlight that to date, most of the biocomputing developments in synthetic biology dealt almost exclusively with genetic material and parts. This highlight clarifies that being confined to this type of approach limits the scope of the potential synthetic biocomputations that can be executed, since a number of important resources are not being utilized [9].

When modeling biocomputers it's also important to distinguish between living biological systems or artificial life, and non-living systems containing biological matter. Human-Centric Functional Modeling is most useful in modeling living biological systems, which are represented as navigating a functional state space while staying within a bounded region within fitness space. Any outcome accomplished by any functional component of a living biological organism or any functional component of an artificial life form can then potentially be represented as a path through some functional state space. In the case of an AGI, for example, reasoning is represented as a path through the space of concepts ("conceptual space") forming the functional state space of the cognitive system. In the case of a General Collective Intelligence, as another example, collective reasoning is represented as a path through the collective conceptual space. In all of these systems of computing, the common element is that every function executed (and therefore every computing function) is a path from one functional state to another. Therefore all functions not only have inputs and outputs, but they also have a "context" defined by all the properties required to specific the current functional state from which the function is being executed. In this sense, all functions of living biological systems are "stateful". Human-Centric Functional Modeling is also very useful in modeling how humans might interact with computers or other non-living systems. 
Non-living biocomputers might also operate through interactions between naturally or synthetically manufactured biomolecules, or between larger naturally or synthetically manufactured microstructures, or even macrostructures. However, such interactions do not require awareness of the state of the system, and therefore might be considered "stateless". As an example of stateless functions, the arithmetic functions (addition, subtraction, multiplication, and division) can be used to navigate the entire number space. But these functions do not require awareness of any state. Computers based on logic gates constructed with such biological substances and structures will not have "awareness" of their functional state.

\section{Related Work}

Other researchers make a similar point as that argued in this paper, namely that biocomputing has specific applications in which it is likely to have an advantage. This paper makes the suggestion that biocomputers are likely to have an advantage in information based computing only for very specific problems. Most of the areas where biocomputing might have some advantage is "physical computation", or computing a path through some physical state space that enables a desired physical configuration to be reached. An example of viewing physical computing as such a change in physical configuration might be finding a way that cells can "compute" a way to grow wood on some substrate shaped as a piece of furniture.

With regards to finding the specific problems where biocomputing might have an inherent advantage, as said by one researcher, "several factors substantially limit the progress in the field. First, there is still no distinct set of particular computational problems that demonstrate a substantial superiority of a biocomputer over modern conventional computational systems. The second basic problem is that computations are slow, being limited by the rates of biochemical reactions" [10]. Other researchers echo this opinion that biocomputers might not likely be competitors to traditional computers in a number of areas, suggesting that the potential applications for biocomputing lie in biosensing, rather than in computation [11].

One area where biologically inspired computing might prevail is Artificial General Intelligence or AGI [1]. As mentioned, the Human-Centric Functional Modelling approach referred to in this paper has been used to define what is believed to be the first model of AGI with the potential capacity for human-like general problem-solving ability. Another area is General Collective Intelligence or GCI [2], where as also mentioned, a model of collaborative computing with the potential for exponentially greater general problem-solving ability (intelligence) than any individual, and also with the potential to solve problems of collective optimization, such as the Sustainable Development Goals, which other forms of computing might lack the capacity to reliably solve. To the author's knowledge the author's own work is the only model that defines the specific mechanisms required for an AGI with the potential capacity for humanlike general problem-solving ability, or the specific mechanisms required for a GCI with the capacity for an exponential increase in intelligence. Because of this, and because these models are so new, all of the existing literature surrounding AGI or GCI from the perspective of Human-Centric Functional Modeling refers to the author's own published work, or pre-prints of the author's unpublished work that are still under review.

\section{Research Questions and Approach}

The research questions addressed in this paper are approached with the method described below: 
Section 4: Research Question Addressed

4.1 Can Human-Centric Functional Modeling be used to represent a subset of biocomputing systems?

4.1 How does representing biocomputing system in terms of Human-Centric Functional Modeling contribute additional insight?

\section{Section 5: Model}

5.1. Computing in terms of stateful operations in a functional state space.

5.2. Computing in terms of stateless operations.

Section 6. Method

6.1. An analysis was performed to assess the requirements for modeling stateful biocomputing. 6.2. An analysis was performed to assess the requirements for modeling stateless biocomputing. 6.3. An analysis was performed to assess the benefits and limitations of stateful biocomputing. 6.4. An analysis was performed to assess the benefits and limitations of stateless biocomputing.

\section{Section 7. Findings}

Section 8. Research Limitations

Section 9. Practical Implications

Section 10. Conclusions

\section{Model}

Any discussion of problems that can be more easily solved through Human-Centric Functional Modeling must begin by understanding the difference between this form of modeling and any alternatives.

\subsection{Computing in Terms of Stateful Operations in a Functional State Space}

Since any process in a given functional domain is represented by execution of a sequence of operations that defines a path through that functional state space, then any outcome accomplished by that path is "computed" by executing those operations. That operation is "stateful" when the choice of which operation is executed depends on the state of the system. Unlike a computer a system of stateful computation such as a human brain changes it's state, perhaps getting tired, and that tiredness changes its operation. Stateful operations can be used to compute outcomes in any functional domain (any domain represented by a functional state space through which a path representing a computation can be defined). This includes both physical domains as well as the information domain represented by the conceptual space.

In Human-Centric Functional Modeling every problem within a functional state space becomes a problem of understanding how to navigate from one point in functional state space to another. In Human-Centric Functional Modeling living systems are represented as having a "collective outcome optimization function" that orchestrates execution of the functional components of the system in a way that maintains the system's fitness to continue to function based on its state. In domains such as homeostasis or reproduction, that collective fitness function might define a path through a functional state space that represents the physical configuration of the system, and in this way "computes" physical outcomes. In the domain of cognition, that collective fitness function is the cognitive awareness process which computes which reasoning process (actively directed navigation through conceptual space) or which understanding process (passively directed navigation through conceptual space) to execute. These reasoning and understanding processes are in turn composed from a minimal set of functions. In defining 
a path through the conceptual space that cognitive awareness process "computes" informational outcomes.

The collective outcome optimization function might operate within a narrow region of functional state space, and therefore be "narrow problem-solving ability", or the collective outcome optimization function might operate within a broad region of functional state space and therefore be general problemsolving ability. A system with narrow problem-solving ability in conceptual space might be considered to be a (problem-solving) tool.

\section{Narrow Problem-Solving Ability}

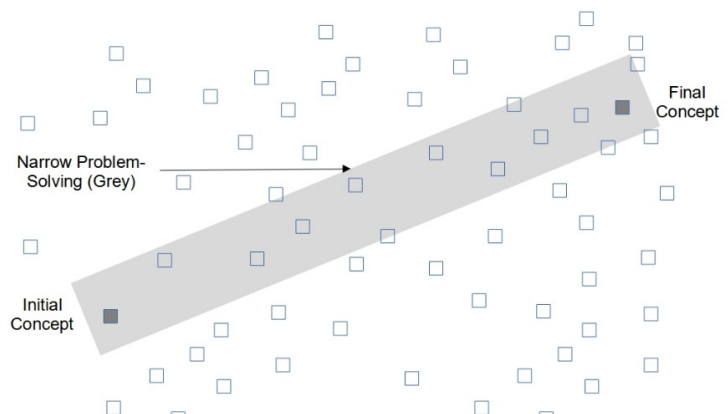

Figure 7: In Human-Centric Functional Modeling a problem is the lack of a path from one concept in conceptual space to another. Narrow problem-solving ability is depicted as the capacity to form such a path (grey band).

A system with general problem-solving ability in conceptual space might be considered to be an independent actor.

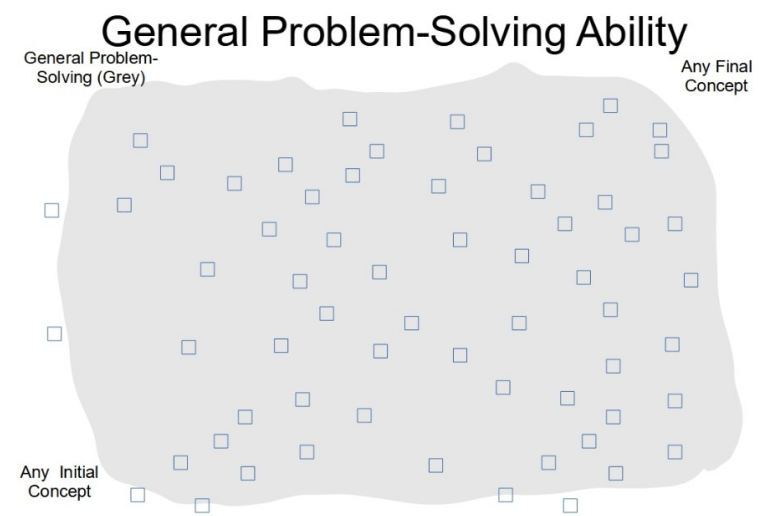

Figure 8: In Human-Centric Functional Modeling general problem-solving ability is depicted as the capacity to potentially form a path defining a solution between any two concepts defining a problem (broad grey region).

This depiction of general problem-solving ability potentially applies not just for information based processing in conceptual space, but for any problem-solving in any other functional state space, such as functional state spaces representing the physical, or metabolic configuration of a biological system. Rather than executing human-defined computing operations such as basic boolean operations, and rather than doing so within logic gates, computing problems might then be defined as the problem of navigating from one point in a functional state space to another point in a functional state space of a biological system. 
The speed and scale of computation in any domain must be limited by the rate at which a volume in functional state space can be explored by the system. In the information domain, with a stateful system like human cognition, this capacity to navigate a volume of conceptual space per unit time is equated here with general problem-solving ability (intelligence). No clear way is currently known to exponentially scale the processing power of any such individual system in order to gain the power to solve significantly different problems. However, Human-Centric Functional Modeling suggests that combining such individual systems to create a potentially much larger collective functional states space that the collective might move through at far greater speed and scale than any individual functional component is capable of is an existing and proven pattern in nature for exponentially increasing that processing power. At least three possibilities for leveraging this pattern come to mind. One possibility is virtualizing such systems, executing each virtual system using stateless computing, and combining the virtual systems into a collective virtual system also executed using stateless computing, that can be used to compute outcomes of collective optimization problems where the individual systems are not reliably capable of doing so. In the cognitive domain this is equivalent to combining a number of AGIs into a General Collective Intelligence. The second possibility is defining interfaces between each such system and the collective virtual system. In the cognitive domain this is equivalent to using a General Collective Intelligence platform to combine individual people or intelligent agents working on behalf of each individual, into a single collective intelligence. The third is architecting a collective system in the same domain. In the cognitive domain this is equivalent to genetically engineering humans to have physical interfaces through which they are able to form a General Collective Intelligence.

In physical domains virtualizing a biocomputer would equate to computing the physical evolution of the functional state space representing that system. In the case of the cognitive domain virtualizing the cognitive system as a biocomputer would equate to computing the evolution of of the conceptual space of an Artificial General Intelligence as it executes cognitive processes.

Because a model of human cognition has been defined with Human-Centric Functional Modeling, because this model of cogntion represents all reasoning as a path through its functional state space (the "conceptual space"), and because human cognition can potentially represent all problems involved in information based computing, then if this model of human cognition is valid it stands to reason that all information based computing problems might be represented in terms of a path through a functional state space defined using Human-Centric Functional Modeling as well. Biological computers have been shown to have the capacity for information based processing. The human cognitive system, is one example, but man-made biocomputers capable of much simpler operations have been demonstrated [13]. If the operations that at least one biological computer (the human cognitive system) is capable of are Turing complete, then all computing functions can be implemented. And if all such operations are implemented in terms of some biological structure, then all computing operations can be represented in terms of some biological function, and therefore represented using a functional model.

Each process collectively executed by such a biocomputer might be represented in terms of a path through the collective functional state space, but might also be representable in some higher order functional state space constructed within that functional state space. For example, the conceptual space has been defined as the functional state space of the cognitive system. However, a functional state space might be defined within the conceptual space to represent all of the operations of a blockchain platform [14]. Or a functional state space might be defined within the conceptual space to represent all the operations of an Internet of Things platform [15]. 
Each biocomputer follows a path through the collective functional state space of its functional components. In order to follow this path, the outputs of one processing activity by one functional component must be passed as inputs to the next processing activity executed by another functional component. In order for this computing to be scaled over many functional components, there must be channels for functional components to exchange outputs of processing to be received as inputs to the next functional component. Any process that is collectively executed by a set of functional components might have a number of attributes of processing. For example, one attribute might be the identity of the functional component currently being executed. A virtual platform with the capacity to orchestrate the collective execution of processes by such functional components must be able to virtualize every attribute of such processes so that each functional component can exchange a virtual representation of that attribute. One such platform has been proposed [6].

\subsection{Computing in Terms of Stateless Operations}

It is proposed here that stateless operations can only be used to compute outcomes virtually, since physical systems have state. When "computing" to achieve an actual change in physical configuration, as opposed to virtually computing a change in configuration, in virtual (non-physical) domains. Computing logic is stateless in the sense that it does not consider the physical state of the computer. Stateless logic in a biocomputer might be accomplished through logic gates representing Boolean operations, or higher order processing units capable of processing more complex instruction sets. The logical operation of such components is well-defined. The potential implementations of such components with biological materials might be unknown, but one requirement for computing in terms of stateless operations is that each function in the biocomputer must at least approximate a stateless one. That is, within the parameters of its normal operation, the components of a stateless biocomputer cannot travel through a functional state space in a way that is bound by stability in fitness space. Just like a computer is not expected to change it's state and get tired, and tiredness is not expected to change the operation of the computer, a stateless biocomputer must also process information independently of its state. If global stability in fitness space and local instability in functional state space is a characteristic of life, a biocomputer must either not be alive, or function as if it is not alive within the parameters that it functions as a biocomputer.

\section{Method}

The research question was addressed using the methods summarized in table 2.

Table 1. Research question and method.

\begin{tabular}{|c|c|}
\hline Research Question & Method \\
\hline $\begin{array}{l}1 \text { Can Human-Centric Functional Modeling be } \\
\text { used to represent a subset of biocomputing } \\
\text { systems? }\end{array}$ & 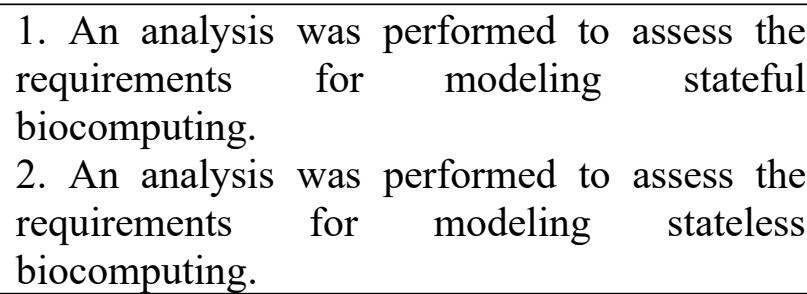 \\
\hline $\begin{array}{l}\text { 2. How does representing biocomputing } \\
\text { system in terms of Human-Centric Functional } \\
\text { Modeling contribute additional insight? }\end{array}$ & $\begin{array}{l}\text { 3. An analysis was performed to assess the } \\
\text { benefits and limitations of stateful } \\
\text { biocomputing. }\end{array}$ \\
\hline
\end{tabular}


4. An analysis was performed to assess the

benefits and limitations of stateless

biocomputing.

\subsection{Requirements for Modeling Stateful Biocomputing}

The requirements for modeling stateful biocomputing are the requirements for modeling logic as a path through any functional state space in the hierarchy of functional state spaces included in the model. For example, in the conceptual space navigated by an AGI, any logic is modelled as a path through conceptual space as a result of executing various functional components of the cognitive system. A complete set of these functional components has been proposed. The conceptual space has only been partially defined however. In order to complete the representation of logic as a network of nodes in a graph of conceptual space, details like the distances between the nodes in the graph still need to be worked out. However, approximations have been made that bypass these details, allowing the approach to be used to model stateful biocomputing even today [5].

\subsection{Requirements for Modeling Stateless Biocomputing}

The requirements for modeling stateless biocomputing are the requirements for modeling logic at any level of abstraction involved in the design of computing systems, such as elementary logic gates within biocomputing hardware, or such as the circuits implementing those logic gates. Since computers exist, implementations for the complete set of these logical elements at every level of abstraction is known.

\subsection{Benefits and Limitations of Stateful Biocomputing}

In representing information based computing, computing of physical configuration, or other biological computing in terms of a path through functional state space that is bound by the requirement for stability of dynamics in fitness space, it becomes clear that the rate at which this dynamical stability can be achieved might limit capacity to scale the rate of executing boolean operations or other stateless computing functions. However, in this modeling approach, the requirement for dynamical stability within a given functional domain puts no limit on the complexity of functional states within that domain. While limits on the complexity of functional components that can be reached over time in the current domain might be created by the homeostasis domain, the growth domain, or other "lower" domains, once those complex functional states are created they might be navigated. This suggests that rather than scaling capacity for information processing using biological computers, scaling capacity for complexity of physical configuration, or scaling complexity in other domains, might be the most effective use of biological computing.

What functions can a biological system have? Functions must be globally stable in fitness space. Global stability requires that the system does what functions to achieve fitness at an outcome rather than adhering to some definition of an outcome from any non-functional perspective. Determination of metrics like time, distance, volume, etc. are made not by any external system of metrics, but instead by outcome. In other words, the time is " $\mathrm{X}$ " when enough time has passed that the time interval has functioned to achieve the targeted outcome. The distance is "Y" when enough distance has been traveled that the travel has functioned to achieve the targeted outcome.

Artificial functions have inputs and outputs that are independent of any context, and therefore defined in an absolute way that is independent of the environment. Biological functions have inputs, outputs, and contexts and act to achieve some outcome that are defined according to their function in the 
environment, that is therefore dependent on the environment. Living biological computing does not rely on stateless non-biological functions such as arithmetic functions, but instead relies on stateful biological functions to navigate functional state space. Rather than trying to create biological computers that implement stateless non-biological functions, a more powerful approach might be to implement biological computers that leverage the capacity of biological systems for complexity, using that capacity to implement more powerful biological functions. For example, rather than attempting to create a set of biological logic gates "grown" from a fungus, where those logic gates might be combined into a digital watch, a more powerful application of biological computing might be to create a fungus that can that can sense subtle changes in the biochemistry of the environment and then biolumininesce to tell us when key events have occurred.

Being globally stable in fitness space, biological processes have a limit to which they can be scaled. Rather than seeing biological computing in terms of boolean operations, a more useful approach would be to see biological computing as carrying out whatever operations in functional state space that are required to achieve an outcome through that navigation of functional state space. For example, some functional state space might be defined in which a sequence of outcomes functions to allow tree cells to grow wood. Another functional state space might be defined to combine individual cognitive systems (individual minds) into a second order cognition (a General Collective Intelligence).

Table 2. Research Findings.

\begin{tabular}{lll}
\hline Biological Computer & Domain Object & Targeted Outcome \\
\hline Modified Tree & [Unknown] & Grow Furniture \\
\hline Modified Cognition & Concept & Second Order Cognition \\
\hline
\end{tabular}

Using current methods in order to build a modified tree cell with the capacity to bind to a substrate and take the shape of that substrate would involve selective breeding as well as trial and error based genetic manipulation. This might take a great many years. Using functional modeling would involve defining functional models of a wide variety of living organisms, and constructing a genome as well as gametes that implement whatever combination of functional components is required. That functional modeling effort might take decades, but once completed, that structure might be completed in whatever amount of time is required to execute gene editing tools capable of operating on plant life, as CRISPR is currently used on animals.

Significantly increasing information computing power through biological computing or through biology inspired computing might involve implementing a second order cognition capable of exchanging meaning rather than information, which is predicted to result in the capacity for an exponential increase in general problem-solving ability over that of an individual human [16]. Further exponential increases might be created by implementing higher order systems of cognition. A General Collective Intelligence or GCI is an artificial system of second order cognition. An Nth order General Collective Intelligence is an artificial system of Nth order cognition. It is unknown whether it is possible for second order or higher order cognition in physical organisms to evolve naturally, or whether such system might only be created through genetic manipulation or other means.

It is proposed here that stateless biocomputation cannot reliably solve collective outcome optimization problems involving collections of functional components. Seeing the functional components of a 
General Collective Intelligence as individual human beings, collective outcome optimization problems include orchestrating massive cooperation in order to maximize collective outcomes. The benefits of higher order systems of cognition like GCI are then the capacity to reliably solve collective optimization problems, which virtually all the existential problems facing mankind might fall into. Living organisms have collective optimization functions in that, for example, the cells in some body part don't tend to spontaneously starve and die due to lack of coordination between the other body parts. The same is not true of human groups, in which some subset of humans are overwhelmingly likely to fall victim to some negative outcome as a result of lack of coordination. For this reason, it might be speculated that currently there might not be any system able to methodically optimize outcomes across an entire population through orchestrating massive cooperation. Therefore there might not be any collective optimization function. As defined within Human-Centric Functional Modeling, a higher order system of cognition such as a GCI must have such a collective optimization function. If having a collective optimization is a property of a higher order cognition, then a higher order system of cognition, might be required for any problems requiring collective optimization to be reliably solvable.

The benefits of higher order systems of cognition like GCI are then the capacity to reliably solve collective optimization problems, which virtually all the existential problems facing mankind might fall into, like achieving collective impact on poverty, access to affordable health care or other Sustainable Development Goals. The limitations of higher order systems of cognition like GCI are that implementation of one by any individual, or orchestrating the cooperation required to collectively implement one, might be problems of "higher order complexity" than any one individual is reliably capable of [17].

\subsection{Benefits and Limitations of Stateless Biocomputing}

Boolean and arithmetic operations might be very efficient and effective for silicon based computing, but might be less effective for biological computing because they likely require translating from more native models, potentially models in terms of functional state spaces. Reframing problems of computing in terms of transitions between functional states is not just a problem of understanding. Changing to a computing system confined to a bounded region in fitness space changes the type of computing operations such a system will be effective at.

Electrical, chemical, physical, or other processes of interaction can occur independently of the state of any system, and therefore can potentially be scaled without limits as long as the required inputs are present and as long as the undesired outputs are removed. Biological processes however, as mentioned occur within some functional component of a biological system and can only be scaled within the limited capacity of the component involved to maintain fitness to function. In other words, while biological functions are inherently stateful, non-biological functions can be stateless. While biological molecules can potentially be synthesized artificially and manufactured at scale, the greater the complexity of those molecules the less the likelihood they can be manufactured at all, or that they can be manufactured at scale. Therefore, the more complicated the structure the less likely it is that processing power can be increased to the massive scales required to compete with non-biological computing technology. As a result, rather than scaling ability to do stateless computing, a biological computer might be more effective at stateful applications. But even though stateless biocomputing is always likely to be less efficient, one likely benefit of stateless computing in a biocomputer would still be complete biodegradeability.

\section{Findings}


The findings are summarized in table 3 below.

Table 3. Research Findings.

\begin{tabular}{ll}
\hline Research Question & Findings \\
\hline $\begin{array}{l}\text { 1 Can Human-Centric Functional Modeling be } \\
\text { used to represent a subset of biocomputing } \\
\text { systems? }\end{array}$ & $\begin{array}{l}\text { The answer appears to be yes. HCFM can be } \\
\text { used to model stateful biocomputers. }\end{array}$ \\
\hline $\begin{array}{l}\text { 2. How does representing biocomputing system } \\
\text { in terms of Human-Centric Functional Modeling } \\
\text { contribute additional insight? }\end{array}$ & $\begin{array}{l}\text { HCFM has the potential to radically } \\
\text { accelerate development of biocomputers, and } \\
\text { to focus effort on applications with the } \\
\text { greatest potential for impact. }\end{array}$ \\
\hline
\end{tabular}

Human-Centric Functional Modeling can potentially be used to represent the path of any biological system through functional state space, and that path through functional state space represents a computing operation. When the computing operation being performed by a biocomputer is a stateful operation, and therefore an operation in a functional state space, it might be directly represented through Human-Centric Functional Modeling. When the computing operation being performed by a biocomputer is a stateless operation, Human-Centric Functional Modeling is only useful for representing the human interaction with that computing system.

The additional insight that Human-Centric Functional Modeling lends to biocomputing is firstly suggesting that biocomputing might have an advantage solving "native" problems in each problemsolving domain, whether solving information problems, or solving problems of physical configuration. Secondly, Human-Centric Functional Modeling creates the potential to optimize collective outcomes among functional components in any given functional domain. In the domain of cognition this includes creating the potential to radically accelerate development of biocomputers through defining a single functional model that might be used to organize all biocomputing research efforts into effectively a single collective effort. While other efforts seek to create efficiencies that might accelerate research and increase research impact, such as through maximizing the distribution and use of developed software within the scientific community given that journals and conferences often require the availability of software and source code [12], maximizing all efficiencies across all researchers requires some collective optimization function, and therefore likely a system of second order cognition such as General Collective Intelligence.

\section{Research Limitations}

It has been said that the majority of accepted papers in biocomputing describe new software approaches to relevant biological problems [12]. In other words, solving application problems rather than solving modeling problems might generally be a greater area of focus in biocomputing.

Human-Centric Functional Modeling has so far been used to define the functions in a functional state space for the cognitive system (the conceptual space). However, as mentioned, the distances between concepts and other properties of the conceptual space that are required to fully model reasoning as a computing process in the functional state space have not yet been elaborated. Since all functional state spaces are expected to share common properties, such as each region in functional state space being fully self-describing, solving these problems for the conceptual space might solve them for all other 
functional state spaces. At the same time, not having solved these problems in conceptual space means that the distances between states and other properties of any functional state space that are required to fully model physical, metabolic, or other transformations as computing processes in the applicable functional state space have not yet been elaborated also. In addition, for most other domains, such as homeostasis, or reproduction, a minimal set of functions capable of spanning all processes in the domain have not been defined. With these limitations, conclusions about which applications biocomputing is most suited for have been deduced in this paper using assumptions that are partly speculative, as opposed to through simulations or through mathematical proofs.

In addition, if the focus of biocomputing is solving applied problems, rather than solving the more abstract problem of defining a single framework for modeling, it might take some effort to build an audience for a method such as Human-Centric Functional Modeling, regardless of any potential to accelerate research and increase research impact.

\section{Practical Implications}

As a biologically inspired system of computing, General Collective Intelligence or GCI has the potential to exponentially increase the general problem-solving ability (intelligence) of groups. GCI also provides an example of how functional components in any domain represented using Human-Centric Functional Modeling might be combined into a collective system with far greater general problem-solving ability in that domain. Furthermore, the platform used to orchestrate cooperation between humans in a GCI might also be used to orchestrate cooperation between virtual models of individual system in each domain, in order to compute outcomes of collective systems in that domain. So in any domain in which a biocomputer might be defined, and for which a virtual model of that biocomputer might be used to emulate computation, that same platform might be used to emulate collective computation.

A GCI is intended to execute self-organizing processes that enable groups to organize into potentially massive networks of cooperation that can target any impact, that can radically increase the probability of achieving that impact, that can radically increase the magnitude of that impact, and that can create sufficient value to make that impact self-sustaining. This approach allows us to see that nature has already solved the problems of sustainability, poverty, universal access to health care, and other existential challenges human beings face. Nature targets outcomes through self-assembling processes that grow, evolve, or become more fit in other adaptive domains, so life can find the resources to feed its own increase in ability to target those outcomes from resources that are already there in the environment. All these processes that nature spent billions of years researching and perfecting are modelled and replicated within GCI. For example, rather than non-profits competing for donor funding, a GCI might organize cooperation between thousands of NGOs to radically increase social, economic, or other impacts per dollar of program funds so programs are self-funding once launched. Rather than developing new networks of cooperation a GCI might use an evolution algorithm, or use a reproduction or other adaptive algorithm, to adapt an existing network. A conceptual case study suggests that increasing social impact per dollar by a thousand fold might be reliably achievable. This implementation of biologically inspired computing represents an increase in computing ability that is profoundly significant, because if so, then poverty, the SDGs and other suffering have been solved.

From the perspective of HCFM, human cognition is able to exchange meaning internally between its functional components. A second order cognition must also have the capacity to exchange this internal representation of meaning between instances of first order cognition. Within an individual organism this implies multiple brains. But lacking the capacity to also multiply informational inputs, the usefulness of 
multiple brains connected by a General Collective Intelligence might be limited as far as information based computing. Among the organisms believed to potentially have multiple brains, such as the octopus [19], those brains might provide the capacity to "compute" transformation of the body into a bewildering variety of textures, patterns, and positions.

Within a collection of organisms of a single species GCI requires a new level of connectivity in order to provide the capacity to exchange entire regions of graphs representing the meaning contained in a given region of conceptual space for an individual, as opposed to simply just exchanging information that must be interpreted. An Nth order cognition involving multiple species implies still greater levels of connectivity in order to extend that exchange to those multiple species. The level of connectivity required to do so at global scale would require massively interconnected and relatively static structures with the capacity to manipulate their interaction with a wide variety of other more mobile creatures. In other words, it might require trees that could interface with animals. A physical or virtual Nth order system of cognition is one approach to achieving collective optimization of outcomes across all species.

The evolutionary path to a physical second order or higher order cognition involving multiple instances of one or more species is hard to imagine. In order to interface with animals and trees it would likely need to influence the development of both. In order to influence their development internally it would likely have to infect them. In order to have the capacity to infect all animals and to convey some fitness advantage in communication it would need to interact with their nervous systems. Fungi fit many of these requirements, but not all within the same species of fungi and not for all species of animal. Fungi like Cordyceps infect certain insects and interact with their nervous systems, but rather than conveying a fitness advantage they might be fatal. Other cordyceps fungi might enjoy a beneficial symbiosis, such as the relationship of Ophiocordyceps with the Cicadas that act as their hosts [18]. Yet other fungi infect plants. But a single fungi that does all these things likely does not occur naturally.

There are at least two ways such evolution might occur. One is a global extinction event that opens up a niche in the environment for organisms that gain greater adaptability through rudimentary second order communication achieved via a third party vector like a fungus that could be spread to other of their species. These organisms might then differentiate into a great many other species. The second is through artificial means such as genetic engineering. Of course, it's hard to think of few things more dangerous than developing a fungus that might infect the nervous systems of potentially all living creatures on earth in order to create some connection mechanism between them. An artificial system of General Collective Intelligence is far safer and more reliably.

\section{Conclusions}

Stateless functions in a biocomputing system can be deployed at unlimited scale. Stateful functions can be deployed to the limit of the system to maintain fitness to execute all of its functions. The type of operations that are likely to be more effective in a biocomputer might likely be stateful operations to build biodegradable products, rather than information-based computing. However, one type of information based biologically inspired computing that is likely to be greatly beneficial is General Collective Intelligence, or higher order General Collective Intelligence, since it creates the potential ability to solve problems that involve optimizing collective outcomes, where those problems can't reliably be solved by artificial computing methods. Since this includes every existential challenge facing mankind, whether poverty, or the other sustainable development goals, and since it involves significantly increasing impact in virtually every area of research, whether physics, economics, or medicine, this implementation of biocomputing might be the most important of all. Furthermore, because the features required in a platform able to model the execution of functional models in any domain might be the 
same, implementing a General Collective Intelligence platform might have direct implications in all types of biocomputing, whether information based, metabolic based, or physical configuration based.

\section{References}

[1] Williams A.E. (2020) A Model for Artificial General Intelligence. In: Goertzel B., Panov A., Potapov A., Yampolskiy R. (eds) Artificial General Intelligence. AGI 2020. Lecture Notes in Computer Science, vol 12177. Springer, Cham. https://doi.org/10.1007/978-3-030-52152-3 38

[2] The Relationship Between Collective Intelligence and One Model of General Collective Intelligence, Andy E. Williams, Computational Collective Intelligence, 11th International Conference, ICCCI 2019, Hendaye, France, September 4-6, 2019, Proceedings, Part II, Pages 589-600

[3] Williams, A. E. (2020, April 27). Defining Functional Models of Artificial Intelligence Solutions to Create a Library that an Artificial General Intelligence can use to Increase General Problem Solving Ability. https://doi.org/10.31730/osf.io/hpzb7

[4] Williams, A. E. (2020, August 19). Defining Functional Models of Collective Intelligence Solutions to Create a Library a General Collective Intelligence can use to Increase General Problem Solving Ability. https://doi.org/10.31730/osf.io/q75rv

[5] Williams, A. E. (2021, January 11). Approximating an Artificial General Intelligence or a General Collective Intelligence. https://doi.org/10.31730/osf.io/zsbfe

[6] Williams, A. E. (n.d.). The Peer to Peer Social Fabric as a Platform for General Collective Intelligence. Retrieved from osf.io/preprints/africarxiv/qbxfr

[7] Adleman, L. M. (1994). Molecular computation of solutions to combinatorial problems. Science 266, 1021-1024. doi: 10.1126/science.7973651

[8] Kendon Viv, Sebald Angelika and Stepney Susan 2015Heterotic computing: past, present and future, Phil. Trans. R. Soc. A.37320140225, http://doi.org/10.1098/rsta.2014.0225

[9] Goñi-Moreno A and Nikel PI (2019) High-Performance Biocomputing in Synthetic BiologyIntegrated Transcriptional and Metabolic Circuits. Front. Bioeng. Biotechnol. 7:40. doi:

10.3389/fbioe. 2019.00040

[10] Gotovtsev, P.M., Kirillova, D.A. \& Vasilov, R.G. Biocomputers: Problems They Solve, State of the Art, and Prospects. Nanotechnol Russia 15, 3-12 (2020). https://doi.org/10.1134/S1995078020010036

[11] Evgeny Katz, Biocomputing — tools, aims, perspectives, Current Opinion in Biotechnology, Volume 34, 2015, Pages 202-208, ISSN 0958-1669, https://doi.org/10.1016/j.copbio.2015.02.011. (http://www.sciencedirect.com/science/article/pii/S0958166915000300)

[12] Packaging Biocomputing Software to Maximize Distribution and Reuse

William S. Bush (), Nicholas Wheeler (), Brett Beaulieu-Jones (), and Christian Darabos ()

Biocomputing 2020. December 2019, 739-742

[13] Katz, E. and Wang, J. (2012). Enzyme Logic Digital Biosensors for Biomedical Applications. In Biomolecular Information Processing, E. Katz (Ed.). https://doi.org/10.1002/9783527645480.ch5

[14] Andy E. Williams, Roberto Moro visconti, Andy E. Williams, The Application of Artificial General Intelligence to the Cognitive Blockchain and the Internet of Value, DOI: 10.13140/RG.2.2.15004.31363 [15] Andy E. Williams, Nenad Petrovic, The Role of Cognitive Computing and Collective Cognitive Computing in the Future Internet, January 2021, DOI: 10.31730/osf.io/qb7sm

[16] Williams, A. E. (2020, July 11). Human Intelligence and General Collective Intelligence as Phase Changes in Animal Intelligence. https://doi.org/10.31234/osf.io/dr8qn

[17] Williams, A. E. (2020, November 11). General Collective Intelligence and the Transition to

Collective Super-Intelligence. https://doi.org/10.31730/osf.io/tacyq

[18] Yu Matsuura, Minoru Moriyama, Piotr Łukasik, Dan Vanderpool, Masahiko Tanahashi, Xian-Ying Meng, John P. McCutcheon, Takema Fukatsu, Recurrent symbiont recruitment from fungal parasites in 
cicadas, Proceedings of the National Academy of Sciences Jun 2018, 115 (26) E5970-E5979; DOI: 10.1073/pnas. 1803245115

[19] Gutnick, Tamar and Zullo, Letizia and Hochner, Binyamin and Kuba, Michael, Octopus Vulgaris Uses Proprioceptive and Tactile Information to Direct The Movement of Its Arms. Available at SSRN: https://ssrn.com/abstract=3565033 or http://dx.doi.org/10.2139/ssrn.3565033 